\title{
I-AUV mechatronics integration for the TRIDENT FP7 project
}

\author{
David Ribas, Pere Ridao, Alessio Turetta, Claudio Melchiorri, Gianluca Palli, José Javier Fernández and \\ Pedro José Sanz
}

\begin{abstract}
Autonomous underwater vehicles (AUVs) are routinely used to survey areas of interest in seas and oceans all over the world. However, those operations requiring intervention capabilities are still reserved to manned submersibles or remotely operated vehicles (ROVs). In the recent years, few research projects have demonstrated the viability of a new type of submersible, the intervention AUV (I-AUV), which can perform underwater missions involving manipulations in a completely autonomous way. The EU FP7 TRIDENT project is one of the most recent examples of such technological concept.

This article describes the different mechatronic components that constitute the I-AUV developed for the TRIDENT project, their hardware and software integration, and the performance of the vehicle during the project trials.
\end{abstract}

Index Terms-IEEEtran, journal, $\mathrm{IT}_{\mathbf{E}} \mathrm{X}$, paper, template.

\section{INTRODUCTION}

$\mathbf{N}$ OWADAYS, the capability of autonomous underwater vehicles (AUVs) is still limited to a small number of tasks, basically consisting on the conduction of surveys to generate detailed maps of the seafloor, or to measure certain parameters in the water column. However, new technological developments, like long-lasting battery systems or more efficient and powerful processors, are making possible to tackle more challenging scenarios traditionally reserved to remotely operated vehicles (ROVs) or manned submersibles. One clear example is that of intervention operations involving dexterous manipulations.

A relevant number of field operations in offshore industries, but also in marine science, rescue operations and defence, need intervention capabilities to perform particular tasks. The traditional approach involves the use of ROVs equipped with powerful robotic arms, although manned submersibles may be used when a direct view of the object manipulation is preferred. The drawbacks of these approaches are multiple, ranging from the limitations and high operation costs associated with the use of large vessels equipped with heavy cranes, dynamic positioning (DP) systems and complex tether management systems (TMSs) in the case of ROVs, to the evident hazard of a human presence on a hostile environment in the case of manned submersibles.

For all these reasons, recently some researchers have started to think about the natural evolution of the intervention ROV;

D. Ribas and P. Ridao are with the Department of Computer Engineering, Universitat de Girona, Spain.

A. Turetta is with Graal Tech SRL and the Center of Integrated Systems for the Marine Environment (ISME), Italy.

C. Melchiorri and G. Palli are with the Department of Electronic, Computer and Systems Science, Università di Bologna, Italy.

J. J. Fernández and P. J. Sanz are with the Department of Computer Science and Engineering, Jaume I University, Spain. the intervention autonomous underwater vehicle (I-AUV). Without the need for a TMS and DP, light I-AUVs could be theoretically operated from economic vessels of opportunity, reducing considerably the overall mission cost. With the fast development of batteries technology, and by removing the operator from the control loop, we can even think about intervention operations lasting for several days, where the ship is only needed for the deployment and recovery operations.

Given the novelty of this field of research, there are still few examples of successful I-AUV implementations. Early works can be dated back to the 90's [1], [2], [3], [4]. However, the first relevant field demonstration is found in the context of the SWIMMER EU project [5]. The proposed concept consisted of an AUV acting as a carrier for an ROV. The AUV was in charge of autonomously navigating to an offshore infrastructure and docking into it to establish a connection with an operator on a base station, allowing a standard ROV operation without need for a heavy tether to the surface. The

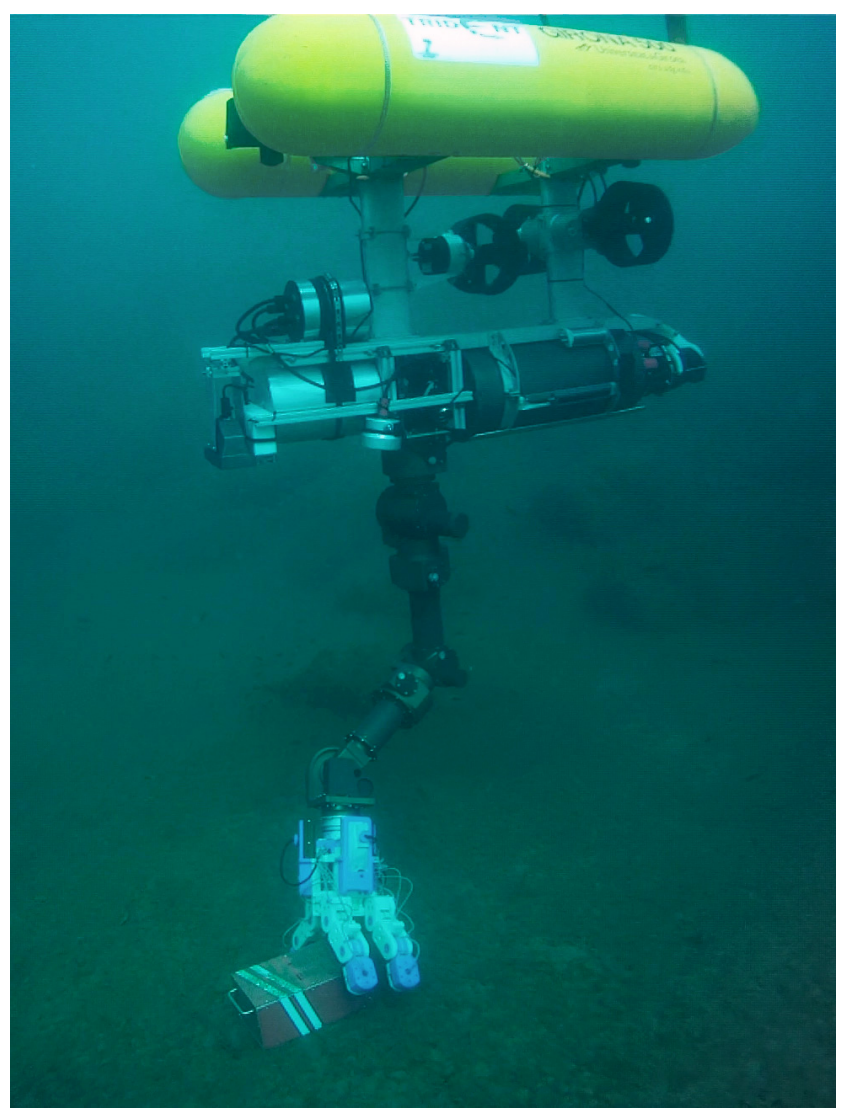

Fig. 1. The integrated I-AUV during sea trials. 
next step towards a fully autonomous intervention system for subsea panels was achieved with the ALIVE project by [6]. It demonstrated the capability of autonomously docking into a ROV-friendly panel using hydraulic grabs. Then, a very simple automata-based manipulation strategy was used to open/close a valve. The guidance of the vehicle to the panel was done using an imaging sonar when far away, and with computer vision when nearby. To the best of the authors' knowledge this was the first time an autonomous intervention was ever made into a subsea panel. Another relevant achievement towards an operational I-AUV came in 2009 as a result of the SAUVIM project [7], During its final demonstration tests, the SAUVIM vehicle was capable of finding and navigating towards an object marked with artificial landmarks and whose position was roughly known a priori. The intervention consisted on recovering this object by hooking a cable to it while the vehicle was hovering over the seafloor.

One of the most recent examples towards autonomous intervention is found in the TRIDENT project. The objective of the TRIDENT project was to provide enhanced autonomous intervention capabilities by enabling a team of two vehicles, an autonomous surface craft (ASC), and an I-AUV to execute a search and recovery mission in two phases. In the first phase, the I-AUV is in charge of surveying the area of interest using optical and acoustic sensors, while the ASC navigates in a coordinated way to provide ultra short baseline (USBL) positioning and reliable communications with the I-AUV. After analyzing the acquired data and determining the object to be recovered (a black-box), the team is sent again on a second phase of the mission where the I-AUV is now responsible, with the support from the ASC, of finding the object and recovering it by means of a dexterous manipulation executed in a free floating mode.

This article reports the integration of the different mechatronics systems that constitute the I-AUV developed for the TRIDENT project (see Fig 1). This I-AUV represents the first example of a new breed of lightweight intervention vehicles. With a weight below the $200 \mathrm{~kg}$, the TRIDENT I-AUV is one order of magnitude less than prior systems like those of the SAUVIM (6 Ton) or the ALIVE (3.5 Ton) projects. Its weight and dimensions make possible to deploy the vehicle from small ships, reducing considerably the operational costs. Another key aspect of the TRIDENT I-AUV is the incorporation of an electric arm with 7 degrees of freedom (DOF) equipped with a three-fingered gripper which offers superior dexterity than the jaw grippers previously used on the referred works.

This article is structured as follows. First, a general description of the I-AUV concept is given in Section II. Sections III to $\mathrm{V}$ present the different components that constitute the vehicle, while Section VI provides details on the hardware and software integration. The article ends with the results and conclusions in Sections VII and VIII.

\section{MeChatronics FOR THE FP7 TRIDENT PROJECT}

As previously introduced, one of the main objectives of the TRIDENT project was to build a robotic system to enable freefloating multipurpose manipulation for lightweight underwater vehicles. To achieve that, it was necessary to design and develop a new intervention payload for an already existing AUV platform consisting of a redundant robotic arm endowed with a dexterous hand, but also to provide the system with new software enabling innovative strategies for the coordinated control of the joint AUV-manipulator system. Developing such thing was not a simple task. For that reason, the consortium behind the TRIDENT project decided to approach the problem as a system of systems. That is, dividing the I-AUV into different parts, each functional on their own, which could be independently developed and tested by each one of the partners. During an initial phase, it was necessary to clearly define the specifications of each system, as well as the hardware and software interfaces. Because of that, it was then quite simple to assemble the different mechatronic systems into the TRIDENT I-AUV in the few meetings where all the teams were together.

Hereafter, the principal mechatronic systems composing the TRIDENT I-AUV are described. The first one is the Girona 500, an AUV developed by the University of Girona, which serves as the basis for the I-AUV. Then, the 7 DOF electric arm made by Graal Tech SRL and finally, a commercial force and torque sensor, the JR3, and two end effectors; a three fingered gripper developed by the University of Bologna, and a much simpler one developed by the Jaume I University as a contingency plan for the period while the Bologna gripper was still under development.

\section{THE GIRONA 500 AUV}

The Girona 500 is a reconfigurable AUV of the University of Girona which was designed for a maximum operating depth of up to $500 \mathrm{~m}$. The vehicle is composed of an aluminum frame which supports three torpedo-shaped hulls of $0.3 \mathrm{~m}$ in diameter and $1.5 \mathrm{~m}$ in length as well as other elements like the thrusters. This design offers a good hydrodynamic performance and a large space for housing the equipments while maintaining a compact size which allows to operate the vehicle from small boats. The overall dimensions of the vehicle are $1 \mathrm{~m}$ in height, $1 \mathrm{~m}$ in width, $1.5 \mathrm{~m}$ in length and a weight of less than $200 \mathrm{Kg}$. The two upper hulls, which contain the flotation foam and the electronics housing, are positively buoyant, while the lower one contains the more heavy elements such as the batteries and the payload. This particular arrangement of the components makes the separation between the centre of gravity and the centre of buoyancy about $11 \mathrm{~cm}$, which is significantly more than any typical torpedo shape design. This provides the vehicle with passive stability in pitch and roll, making it suitable for imaging or bathymetric surveys, but also for other tasks requiring an stable platform such as the free-floating manipulation envisioned for the TRIDENT project.

On its standard configuration, the vehicle is equipped with typical navigation sensors such as a Doppler velocity log (DVL), an attitude and heading reference system (AHRS), a pressure gauge and an USBL; but also has basic survey equipment like a profiler sonar, a side scan sonar, a video camera or a sound velocity sensor. However, the most remarkable characteristic of the Girona 500 is its capacity to reconfigure for different tasks. Almost half the volume of the lower hull is 
reserved for installing additional sensors, or mission-specific payloads such as the dexterous manipulator for the TRIDENT project. In order to compensate the weight of the installed payload, free space is located on the top part of the vehicle which can be used to place extra buoyancy modules.

The Girona 500 can also change its propulsion system by setting different thruster configurations. The vehicle can be controlled with a minimum of 3 thrusters. However, more thrusters can be installed to control additional DOF up to a maximum number of 8 thrusters, which allows full redundant control of the 6 DOF. Given the excellent passive stability of the vehicle, only five thrusters were installed during the TRIDENT project trials. Two horizontal thrusters controlled the surge and the heading, one lateral thruster allowed small sway movements and two vertical thrusters provided heave control with extra lift force.

\section{THE UMA ROBOTIC ARM}

One of the main challenges for the mechatronics of the TRIDENT I-AUV was to design a redundant arm with enough dexterity to deal with the uncertainties of autonomous operations taking place in unknown and unstructured environments. Such a concept was quite innovative, as in the field of underwater intervention the usage of redundant manipulators was previously explored just in some pioneering research projects, like AMADEUS and SAUVIM. On the contrary, within industrial applications, where the great majority of underwater manipulation activities have always been remotely driven by operators, simpler arms with a lower number of DOFs are commonly employed, as they are enough for the considered tasks.

The existing distance between the needs of the TRIDENT project and the market demands in terms of underwater manipulation systems pushed Graal Tech to focus their efforts on finding a highly reconfigurable solution. The goal was obtaining a robotic system that could match the TRIDENT specifications but, at the same time, be exploited as widely as possible after the completion of the project.

The result was UMA, the first Underwater Modular Arm, which is currently commercially available from Graal Tech.

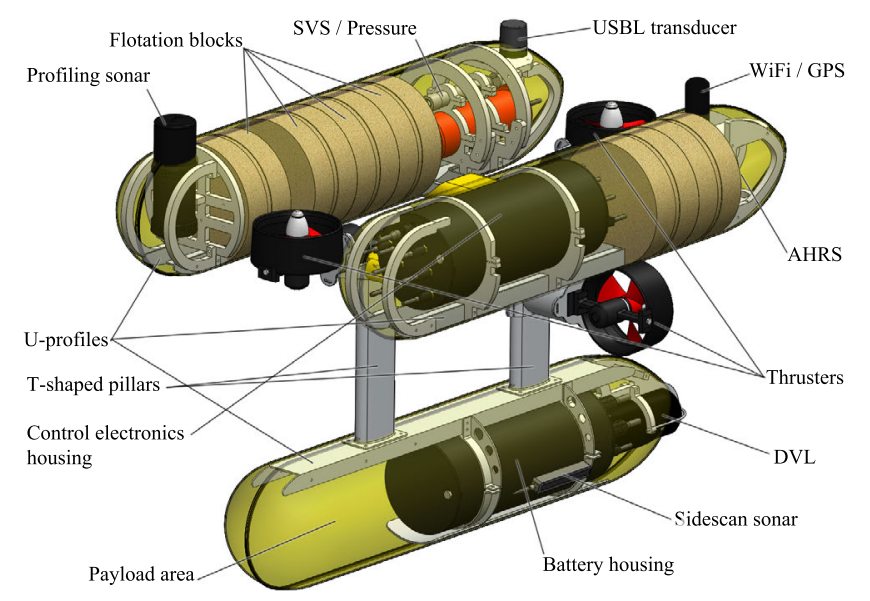

Fig. 2. The Girona 500 AUV.
As the name suggests, UMA is an underwater manipulator, designed by adopting a modular approach. Two kinds of electrically-driven joints are available (respectively with one and two motion axes) together with a set of links for connecting the joints. By varying the basic modules (joints and links) and/or their number, or the way they are interconnected, different kinematic configurations can be obtained. Inside every joint, an embedded dedicated motion controller board provides the low-level position and speed control functionalities for every motor. All the boards are interconnected through a common CAN-bus line, providing a global interface for sending commands and receiving feedback from joints at a rate of up to $200 \mathrm{~Hz}$. The presence of a common data bus significantly reduces the number of electric wirings running inside the arm, thus further facilitating the operations concerning the insertion or removal of a joint. Indeed, on both sides of every joint, just a pair of electric connectors, respectively providing power and data communication lines, are necessary.

A second type of connector, of hydraulic nature, is present on both the terminating interfaces of every joint for connecting them through hydraulic hoses. In this way the presence of a single hydraulic circuit running inside the whole arm, from base to wrist, allows to easily fill (or empty) each joint with oil. The usage of oil is not strictly necessary if the arm is operated at small depths, since the sealing system on each joint is enough to guarantee waterproofness. However, at higher depths $(40 \mathrm{~m}-100 \mathrm{~m})$, the usage of oil is recommended to avoid the risk of water ingress. To this aim it is also mandatory the use of a pressure compensator for maintaining the internal oil pressure slightly above ambient pressure.

Within marine applications two common materials are employed for realizing structural parts: inox steel (AISI 316) and aluminum. Inox offers better properties in terms of resistance against sea-water corrosion, but it is significantly heavier (around 3 times more) than aluminum. Therefore, in order to reduce the weight, the arm has been realized with an aluminum alloy (ERGAL) typically used for aeronautic applications. Further it has been properly treated with a process of hard anodizing, which increases the surface hardness and protects against sea-water.

Both families of joints are driven by brushless DC motors, which, on the contrary of brushed motors, can operate while submerged in oil. All the motors are from Faulhaber Gmbh, the German company manufacturing also the embedded servo boards. For every joint the specific model was selected by taking into account the size and the required torque and speed specifications. Three types of motors (from 2444, 3564 and 4490 series) have been selected for actuating the 7 joints of the TRIDENT arm. The servo boards are all from the MCBL300x series and provide both speed and position control functionalities.

Inside every joint, the mechanical gearing system consists of a Harmonic Drive Component Set, which is a highly stiff and accurate device with zero-backlash. The usage of Harmonic Drive gearing systems is quite common in robotic applications, as they can provide high reduction factors despite their small size. As a consequence, very often the employment of a Harmonic Drive device eliminates the need for additional gearing 


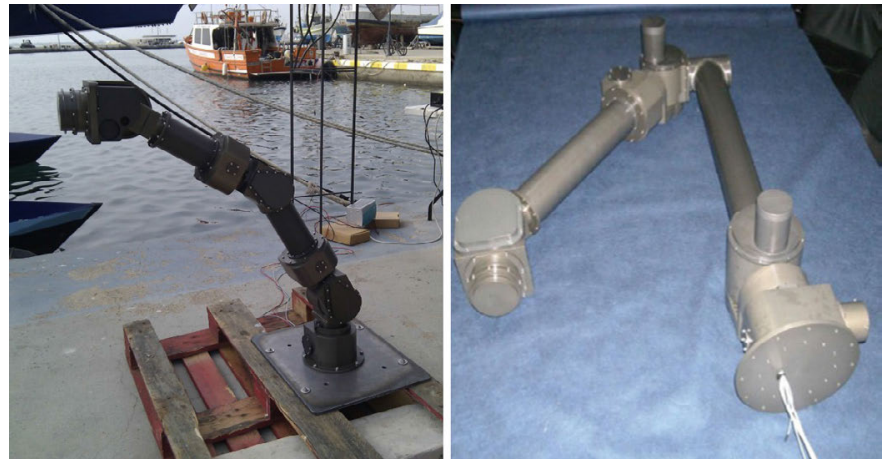

Fig. 3. Different configurations of the arm are possible because of its modular conception. On the left, the arm with $7 \mathrm{DOF}$, on the right, with $6 \mathrm{DOF}$.

elements, thus reducing significantly design and production costs, and leading to very compact systems. The HFUS-2UH series have been selected for the UMA robot, as the gearboxes from this family are completely sealed thus allowing the usage with oil. They also feature a large central hollow shaft (with a diameter of up to $30 \%$ of the outside diameter), allowing an easier routing of the cables through the joint and, therefore, contributing to limit the size of the joints.

To protect the joints from possible water leakages, all the elements of the external covers that are assembled together have conventional O-ring-based sealing elements inserted between the matching surfaces. On the other hand, the dynamic sealing between the moving parts relies on lip rings. They are flexible sealing elements, but with a peculiar "c"-shaped crosssection instead of the circular one characteristic of O-rings. As O-rings, they are mounted in the interfacing region between the coupling parts. However, the direction they are mounted is not irrelevant. Indeed, due to its concave cross-section, the lip ring can be always considered as "filled" by one fluid, be it the external water (if the open part of the "c" is directed outside), or the internal air (or oil) in the other case. For this reason, and to increase the protection against water leakages, whenever the arm is filled by oil and a pressure compensator is used, the lip rings have to be mounted with the open part of the "c" looking inside, in a way that the oil pressure (being always higher than the external one due to the compensator) exerts a force that pushes the lips against their contact points, thus further contributing to improve the sealing.

\section{END EFFECTORS}

This section introduces the two different end effectors developed for the TRIDENT project plus a commercial forcetorque sensor that can be optionally installed between the arm and the hand to provide enhanced sensitivity during the manipulations.

\section{A. The JR3 force and torque sensor}

An underwater 6 DOF force-torque sensor has been designed and manufactured by the JR3 Inc. company using as a basis the ground version of the same sensor, and following the specifications from the Jaume I University. The sensor is contained inside a cylindrical housing made of aluminum and designed to withstand a maximum depth of $50 \mathrm{~m}$. The internals are kept at atmospheric pressure and the sealing is achieved by means of O-rings. Additional O-rings are required to seal the sensor faces with the arm and hand mating plates. A 20 $\mathrm{mm}$ diameter hole placed along the principal axis of the sensor allows to pass cables between the arm and the hand following the mechanical interface specified by ISO9409-1-A 50.

The housing contains analog and digital electronic systems. Foil strain gages bonded to multiple internal load-bearing elements sense the loads exerted on the sensor. The strain gage signals are amplified and combined to produce analog representations of the forces along three orthogonal axes (x,y and $\mathrm{z}$ ) as well as the moments or torques about those same axes. To accurately sense loads of about $20 \mathrm{~N}$ at a moment arm of $250 \mathrm{~mm}$, the sensor has $100 \mathrm{~N}$ mechanical full scale. The sensor has internal electronics with analog output to avoid the use of a custom digital receiver card. Interface is through an analog data acquisition board

\section{B. The UJIOne gripper}

The first end effector, the UJIOne, is an electrically actuated 1 DOF gripper whose long jaws were designed taking into account that the main intervention target of the TRIDENT project was the recovery of a black-box mockup. The gripper had to be simple, reliable and robust to serve as a contingency plan for the more complex UNIBO hand that will be described in the following section. It is made in aluminum with a weight of $3.93 \mathrm{Kg}$ in air and $2 \mathrm{Kg}$ in water and an operational depth of $100 \mathrm{~m}$. Its overall dimensions are $479.47 \mathrm{~mm}$ (long) $x 130 \mathrm{~mm}$ (high) x $104.36 \mathrm{~mm}$ (width) when fully closed and $276.33 \mathrm{~mm}$ (l) $\times 130 \mathrm{~mm}(\mathrm{~h}) \times 561.13 \mathrm{~mm}(\mathrm{w})$ when fully open.

The UJIOne is built around a cylindrical housing that encapsulates a servo motor, the control electronics and a DC/DC voltage converter that allows the unit to be powered with 9 to 36 volts. The housing is sealed with O-rings at their end caps. One end cap allows to assemble the UJIOne on the JR3, or directly on the arm, sealing these assemblies with another O-ring. The other end cap supports the jaws and the transmission mechanism which is composed by a Dynamixel AX-F18 servo motor whose shaft is connected to a worm drive. The shaft crosses the end cap through lip ring seal to prevent water ingress. The module 1 worm gear is then meshed with two 40 teeth spur gears having a 40:1 reduction ratio that provides up to $2 \mathrm{Kg}$ of lift force. Then, the gears transmit the rotation to their corresponding crossing shafts which are connected to the jaws. The jaws are composed by four (i.e. two for each jaw) long curved fingers that allow grasping large objects. When completely folded, the fingers can achieve envelope grasps of small objects, and even execute hooking manipulations given their slim design. Finally, the fingers can also be equipped with FlexiForce tactile and strain gauge force sensors that are sealed with self-amalgamating tape that also provides a non-slipping surface to help grasping.

The UJIOne has an USB interface for the communications between the gripper's microcontroller (an Arduino Mega 2560) and the high level controller from the vehicle. The microcontroller is in charge of transmitting the signals from the JR3 


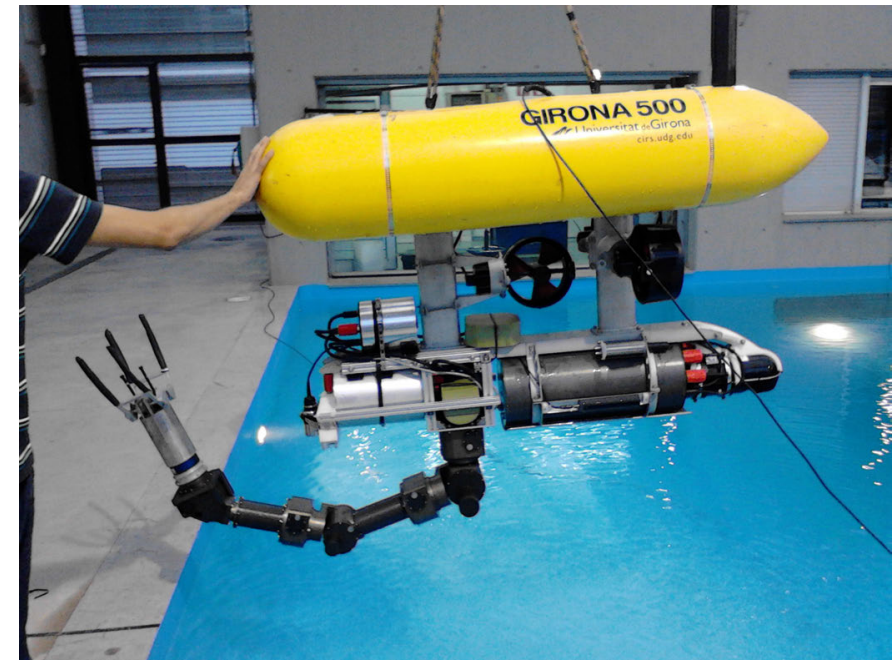

Fig. 4. The I-AUV during the first integration trials at the water tank in the Underwater Robotics Research Center of the University of Girona. The UJIOne gripper can be seen mounted at the end of the UMA. The JR3 sensor is the thin dark band at the wrist of the gripper.

and tactile sensors, but also to actuate the servo according to the position, velocity or load setpoints commanded from the high level control.

\section{The UNIBO gripper}

The UNIBO gripper developed by the University of Bologna is characterized by a large workspace and a higher dexterity than other waterproof end effectors previously reported on the bibliography. Fig. 5 (left) shows a schematic view of the gripper kinematics. The gripper presents three fingers, one named $\mathrm{T}$, which can be intended as an opposable thumb, and two identical fingers named FR and FL (right and left finger respectively). This kinematic configuration is clearly inspired by the famous Barret Hand [8], that represents a suitable tradeoff between hand functionality and design simplicity.

To reduce the weight, $\mathrm{ABS}$ plastic has been largely used for mechanical parts manufacturing, whereas anodized aluminum have been adopted for the metallic parts to prevent corrosion. The overall weight in air is about $4.5 \mathrm{~kg}$, while in water it is about $1 \mathrm{~kg}$. All the gripper joints are of revolute type with Teflon bushings for reducing friction and to prevent corrosion in the sea environment. The thumb has just two links; the proximal link, connected to the palm by a revolute joint (proximal joint) with a rotational axis parallel to the palm plane, and a distal link connected to the proximal link by a revolute joint (distal joint) whose rotational axis is also parallel to the palm plane. The FR and FL fingers differ from the thumb in the way they are connected to the palm. In this case, an additional joint (palm joint) with a rotational axis perpendicular to the palm plane is introduced between the palm and the proximal link, allowing the rotation of the whole finger with respect to the palm axis. This arrangement allows performing both parallel grasps as well as precision grasps, by means of opposition of the fingertips.

Only 3 (identical) motors are used for the actuation. Therefore, couplings among the joints are present. These couplings are implemented in a very simple way by connecting in parallel the cable driving system of the three joint groups (i.e. distal, proximal and palm joints) to the same motor. Referring to Fig. 5 (left), the angle $\alpha$ (distal joint angle), whose range is [90 240] deg, is controlled by one single motor for all the three fingers at once. The same happens for the angle $\beta$ (proximal joint angle), whose range is [30 $180] \mathrm{deg}$, and the angle $\gamma$ (palm joint angle) whose range is [90 150] deg. Although only three motors are used, different configurations can be achieved, allowing a potential of many types of grasps on a great variety of objects, both in force and in form closure. As a matter of fact, the gripper is able to grasp objects up to $300 \mathrm{~mm}$ in diameter. Some significant finger postures and grasp configurations are reported in Fig. 5 (right). The actuation system of the gripper is based on the Faulhaber brushless motor EN 2250 BX4 CCD SERIES with integrated motion controller and CAN interface. The force applicable by each finger (in different configurations) is about $150 \mathrm{~N}$, which can be considered satisfactory for the typical operations of the TRIDENT project. Moreover, the actuators are non-backdrivable, which allows holding the desired gripper configuration without further supplying power to the motors, even during grasp.

The closed-loop cable transmission of the gripper, whose details are visible in Fig. 6, implements a double-acting actuator [9]. This transmission system has been adopted for several reasons:

- it allows optimizing the motors allocation in terms of required space and weight distribution;

- it represents a simple and convenient way to couple several joints to a single motor in a fixed way, so that the effective number of degrees of freedom is equal to the number of motors;

- it simplifies the transmission chain;

- the sheath-based routing introduces compliance in the transmission, allowing a certain adaptation capability of the gripper during grasp of uncertain and irregular objects.

Specific force/torque sensors have been designed and implemented on each fingertip for controlling the force and the stability of the grasp [10], see Fig. 7 where the CAD of the sensor can be seen. The sensor is based on the modulation of the current flowing through a PhotoDetector (PD) as a consequence of the variation of the relative position of an infrared light source (LED), and in particular, of the angle of view between the optoelectronic components and the length of the optical path. The reader can refer to [11], [12], [13], [14] for a complete analysis of the system. The sensor allows detecting the movements of the fingertip surface with respect to the supporting structure during the contact with the grasped object, allowing the estimation of both the forces and the torques applied to the fingertip surface.

The communication between the control system, the motors and the sensors of both the arm and the gripper is implemented through a 2-wire CAN bus for the maximum design simplification. 

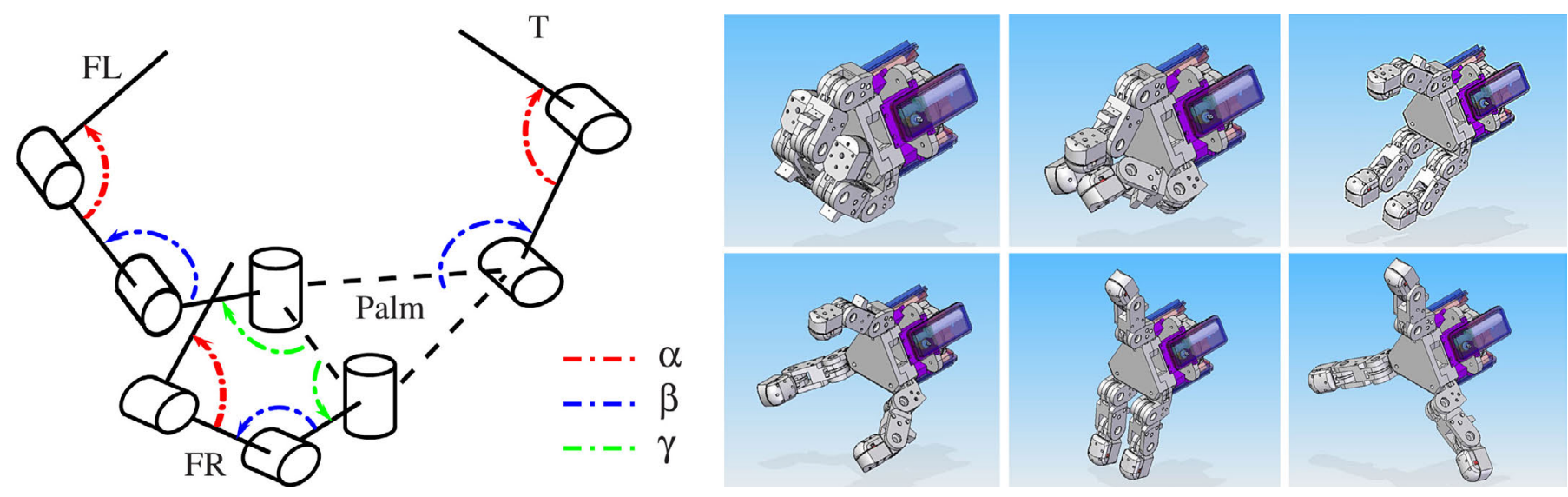

Fig. 5. The kinematic structure of the UNIBO gripper (left) and some CAD examples of kinematic configurations (right).
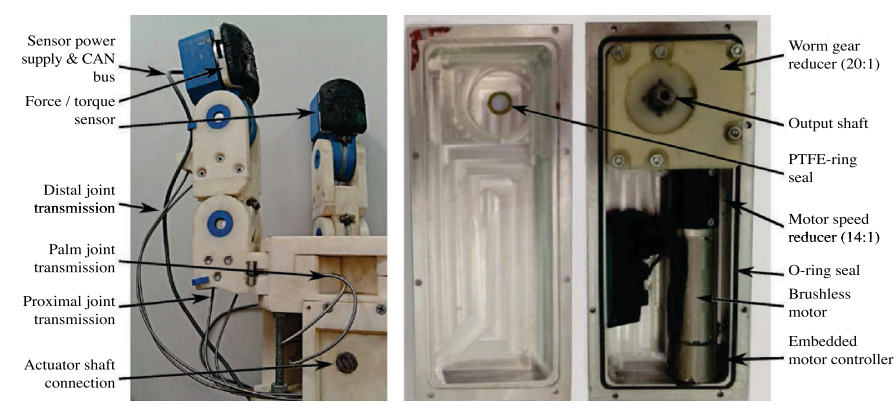

Fig. 6. Detailed view of the cable transmission system.

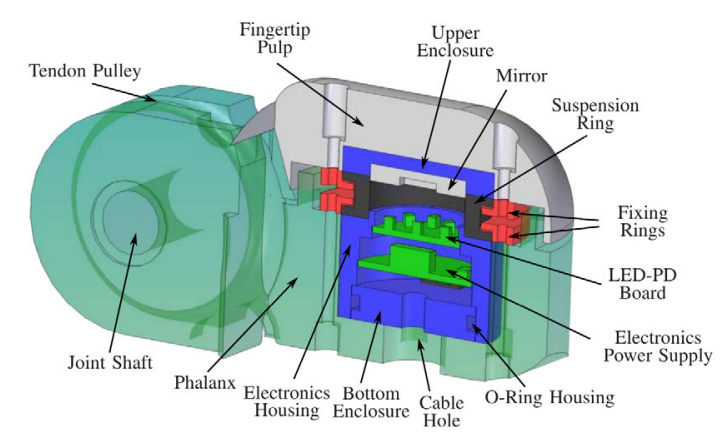

Fig. 7. Internal view of the fingertip force/torque sensor.

\section{HARDWARE AND SOFTWARE INTEGRATION}

As commented, the TRIDENT I-AUV concept uses the Girona 500 AUV as base platform, with the rest of the elements mounted on its payload area. Given the weight of the payload, it needs to be mechanically interfaced with the aluminum backbone of the vehicle's lower hull. The vehicle also provides power and communications to the payload systems (see Figure 8). For this particular implementation, an Ethernet connection was set up between the Girona 500 control computer and the ones in charge of the vision system and the manipulation control. On its standard configuration with the UNIBO gripper, the end effector and the different modules of the UMA arm were connected with the manipulation control computer through a CAN-bus line. The JR3 sensor was directly interfaced with the manipulation control computer by routing its analog signals though the internal cavity of the arm (a similar strategy is adopted for the USB interface of the UJIOne when this is mounted). Given the energy requirements of the manipulator system, direct access to the vehicle batteries was provided. Individual power regulators were installed inside the cylinders of the vision and manipulator control computers to provide the required voltages to the slaved systems.

Regarding the software architecture of the I-AUV, it is based on the component-oriented layer-based architecture for autonomy (COLA2) implemented on the Girona 500 AUV [15]. This architecture is comprised of independent components, each one responsible of a specific task, which are grouped in layers according to their role. The main layers are: the navigation layer, which contains the drivers for all the navigation sensors and the localization filter used to estimate the vehicle's pose and velocity; the control layer, which includes low-level (i.e. pose, velocity and force controllers) and high-level (i.e. teleoperation, trajectory following, mission control, etc.) controllers; the perception layer where all the exteroceptive sensor drivers are; and the detection layer that groups all the detection algorithms. Since the architecture has been implemented using the Robot Operative System (ROS) middleware, it has been straightforward to add new components to control the extra manipulator/end-effector devices and their functionalities. A new layer named manipulator has been added to be in charge of the task-level control [16]. The goal of this layer is to plan and supervise the intervention task, as well as to execute a high-level sensor-based closed loop control of the end-effector. Its outputs are end-effector velocity requests. Also, an underwarer vehicle-manipulator system (UVMS) scheme that jointly controls the AUV and the manipulator has been developed and incorporated as part of the control layer [17]. With this new scheme it is possible to control the UVMS by directly requesting poses or velocities for the end-effector. Figure 9 shows the main layers presented here as well as the basic information flow between them.

\section{EXPERIMENTAL RESUlTS}

According the planning agreed by the TRIDENT consortium, the different teams involved on the development of new 


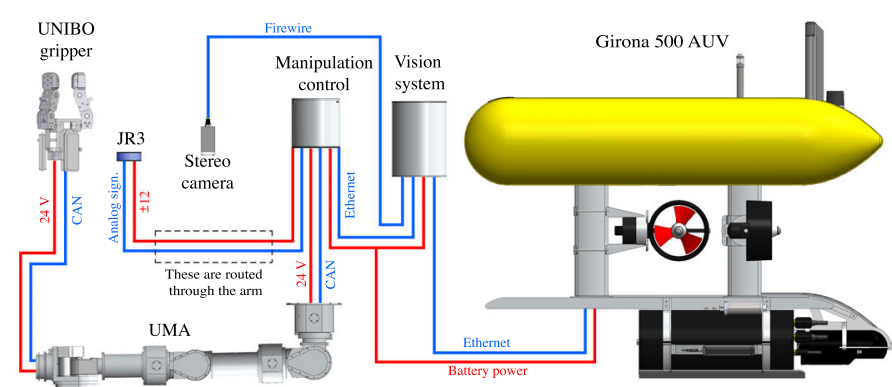

Fig. 8. Schematic showing the connections between the different subsystems

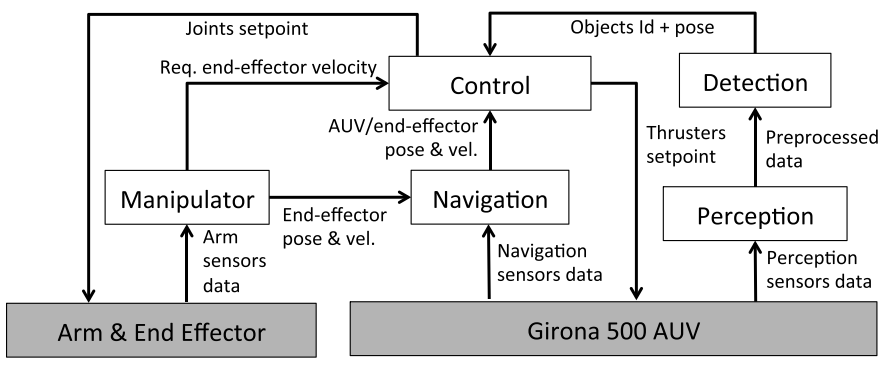

Fig. 9. The layers composing the I-AUV software architecture

mechatronic systems had to have them finished by the end of the second year (of a three years project). This meant that just one last year was devoted to refining the operation of the fully integrated I-AUV. Although the software for the different mechatronics systems, as well as for the fully integrated vehicle, was extensively tested through hardware in the loop (HIL) simulation, it was not until the spring of 2012 that the TRIDENT I-AUV came to life during the first integration tests that took place at the Underwater Robotics Research Center of the University of Girona (see Fig. 4). The vehicle, arm and end effectors were put to test with the black-box recovery benchmark scenario envisioned for the TRIDENT project (see the video in [18]). The I-AUV was capable of completing the task although some flaws were detected, particularly on the design of the complex three-fingered gripper, which forced a second iteration. The final version of the vehicle was tested at the end of 2012 summer during a second set of trials, again at the water tank. The success of those trials led some time after to the final demonstration of the concept in a more realistic scenario in a harbor at Port de Sóller (Spain), where despite the bad visibility conditions, the I-AUV was again capable to successfully demonstrate the task (see Fig. 1, and also the video in [19]).

\section{CONCLUSIONS}

This article has presented the different mechatronic systems that constitute the TRIDENT I-AUV, their hardware and software integration and the experimental testing which led to the successful demonstration of the light intervention capabilities envisioned in the TRIDENT project. The development of the I-AUV was challenging because of the complexity of the systems involved, but also because of the short integration time. From the hardware point of view, the modular conception of the system, and a clear definition of the interfaces were crucial for a simple integration. Likewise, the adoption of the ROS middleware for the software architecture allowed the development of the required nodes independently and later, an straightforward integration. Another important aspect, which played an important role, was the adoption a clear experimental methodology based on three sequential steps: 1) Hardware in the loop simulation (HIL); 2) water tank testing and 3) Sea trials.

\section{ACKNOWLEDGMENT}

This work was supported by the TRIDENT EU FP7- Project under the Grant agreement No: ICT-248497.

\section{REFERENCES}

[1] H. H. Wang, S. M. Rock, and M. J. Lee, "Experiments in automatic retrieval of underwater objects with an auv," in OCEANS'95. MTS/IEEE. Challenges of Our Changing Global Environment. Conference Proceedings., vol. 1. IEEE, 1995, pp. 366-373.

[2] S. Choi, G. Takashige, and J. Yuh, "Experimental study on an underwater robotic vehicle: Odin," in Autonomous Underwater Vehicle Technology, 1994. AUV'94., Proceedings of the 1994 Symposium on. IEEE, 1994, pp. 79-84.

[3] V. Rigaud, E. Coste-Maniere, M. Aldon, P. Probert, M. Perrier, P. Rives, D. Simon, D. Lang, J. Kiener, A. Casal et al., "Union: underwater intelligent operation and navigation," Robotics \& Automation Magazine, IEEE, vol. 5, no. 1, pp. 25-35, 1998.

[4] D. M. Lane, J. B. C. Davies, G. Casalino, G. Bartolini, G. Cannata, G. Veruggio, M. Canals, C. Smith, D. O'Brien, M. Pickett et al., "Amadeus: advanced manipulation for deep underwater sampling," Robotics \& Automation Magazine, IEEE, vol. 4, no. 4, pp. 34-45, 1997.

[5] J. Evans, K. Keller, J. Smith, P. Marty, and O. Rigaud, "Docking techniques and evaluation trials of the SWIMMER AUV: an autonomous deployment AUV for work-class ROVs," in OCEANS, 2001. MTS/IEEE Conference and Exhibition, vol. 1, 2001, pp. 520-528.

[6] J. Evans, P. Redmond, C. Plakas, K. Hamilton, and D. Lane, "Autonomous docking for Intervention-AUVs using sonar and video-based real-time 3D pose estimation," in OCEANS, 2003. MTS/IEEE, vol. 4, 2003, pp. 2201-2210.

[7] G. Marani, S. K. Choi, and J. Yuh, "Underwater autonomous manipulation for intervention missions auvs," Ocean Engineering, vol. 36, no. 1, pp. 15-23, 2009.

[8] W. Townsend, "The BarrettHand grasper-programmably flexible part handling and assembly," Industrial Robot: An International Journal, vol. 27 , no. 3, pp. 181-188, 2000.

[9] C. Melchiorri and M. Kaneko, Springer Handbook of Robotics. Springer, 2008, ch. 15 , pp. 345-360.

[10] G. Berselli, M. Piccinini, G. Palli, and G. Vassura, "Engineering design of fluid-filled soft covers for robotic contact interfaces: guidelines, nonlinear modeling, and experimental validation," Robotics, IEEE Transactions on, vol. 27, no. 3, pp. 436-449, 2011.

[11] G. Palli and S. Pirozzi, "Force sensor based on discrete optoelectronic components and compliant frames," Sensors and Actuators A: Physical, vol. 165, no. 2, pp. 239-249, 2011.

[12] — , "Miniaturized optical-based force sensors for tendon-driven robots," in Robotics and Automation (ICRA), 2011 IEEE International Conference on. Shanghai, China: IEEE, 2011, pp. 5344-5349.

[13] _ " "A miniaturized optical force sensor for tendon-driven mechatronic systems: Design and experimental evaluation," Mechatronics, vol. 22, no. 8, pp. 1097-1111, 2012.

[14] - "Optical force sensor for the DEXMART hand twisted string actuation system," Sensors \& Transducers, vol. 148, no. 1, pp. 28-32, 2013.

[15] N. Palomeras, A. El-Fakdi, M. Carreras, and P. Ridao, "COLA2: A Control Architecture for AUVs," IEEE Journal of Oceanic Engineering, vol. 37, no. 4, pp. 695-716, 2012.

[16] M. Prats, A. P. del Pobil, and P. J. Sanz, Robot Physical Interaction through the combination of Vision, Tactile and Force Feedback, ser. Springer Tracts in Advanced Robotics. Springer, 2013, vol. 84. 
[17] G. Casalino, E. Zereik, E. Simetti, S. Torelli, A. Sperinde, and A. Turetta, "Agility for underwater floating manipulation: Task amp; subsystem priority based control strategy," in Intelligent Robots and Systems (IROS), 2012 IEEE/RSJ International Conference on, 2012, pp. 17721779.

[18] D. Ribas, "Trident FP7 project: Arm/hand integration," 2012, [Youtube video, accessed 14-April-2014]. [Online]. Available: http://youtu.be/uGSuJjMafZg

[19] J. J. Fernández, "FP7 TRIDENT final experiments," 2013 , [Youtube video, accessed 14-April-2014]. [Online]. Available: http://youtu.be/iq5ePDKQB1g

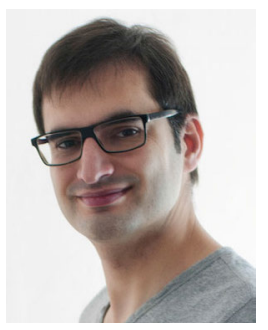

David Ribas received the M.Sc. in Industrial Engineering in 2003 and the Ph.D. in Industrial Engineering in 2008, both from the University of Girona, Spain. His research interests are focused in the development of AUVs and more particularly to the autonomous navigation problem using Simultaneous Localization and Mapping (SLAM) techniques. Currently, he is a researcher in the Department of Computer Engineering at the University of Girona, and a member of the Research Center in Underwater Robotics (CIRS). He is involved in national and European projects about underwater robotics and some technology transference projects about real-time and embedded systems.

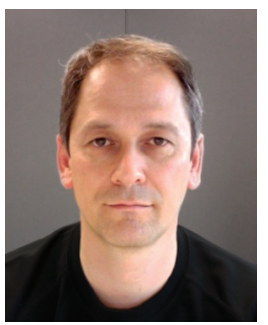

Pere Ridao received the Ms.C. degree in computer science in 1993 from the Technical University of Catalonia, Barcelona, Spain, and the Ph.D. degree in computer engineering in 2001 from the University of Girona, Spain. His research activity is mainly focused on underwater robotics in research topics such as intelligent control architectures, UUV modelling and identification, simulation, navigation, mission control and real-time systems. Currently, he is an associate professor with the Department of Computer Engineering of the University of Girona and the head of the Research Center in Underwater Robotics (CIRS) of Girona. Dr. Ridao is also a member of the IFAC's Technical Committee on Marine Systems, member of the editorial board of Springer's Intelligent Service Robotics journal, secretary of the Spanish OES chapter and also a board member of the Spanish RAS chapter.

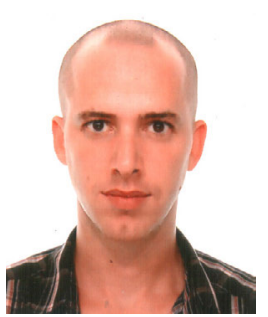

Alessio Turetta graduated in 2000 in Software Engineering from University of Genova, and received its Ph.D. in Robotics in 2005 from the same University. In 2002 he started cooperating with Graal Tech as a control system consultant. During years he consolidated his relationship with the Company, becoming first a Member of the Board of Directors, and then a Partner. In parallel with the Graal Tech experience, since 2005 , he started his scientific and teaching career with the University of Genova and, at the end of 2011, he got a permanent position as Assistant Professor. Today he has no more managerial roles in Graal Tech He just remained a partner of the Company and maintained some technical cooperation opportunities as a control system and embedded system specialist.

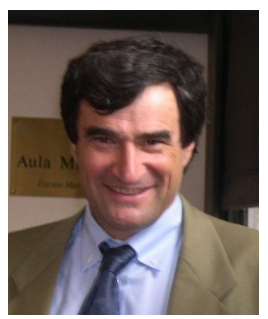

Claudio Melchiorri received the Laurea degree in electrical engineering, in 1985, and the Ph.D. degree, in 1990, both from the University of Bologna, Bologna, Italy. He was the Adjunct Associate in engineering with the Department of Electrical Engineering, University of Florida, Gainesville, in 1988 and a Visiting Scientist with the Artificial Intelligence Laboratory, Massachusetts Institute of Technology, Cambridge, in 1990 and 1991, respectively. Since 1985, he has been with the Department of Electrical Engineering, Computer Science and Systems, University of Bologna, where he has been involved in the field of robotics and automatic control. He is currently the Professor of robotics with the University of Bologna. He has authored or coauthored more than 230 scientific papers in international journals and conferences and 13 books on digital control and robotics. His research interests include dexterous robotic manipulation, haptic interfaces, telemanipulation systems, advanced sensors, and the modeling and control of complex dynamical systems.

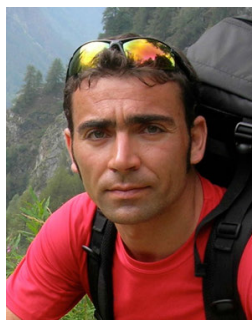

Gianluca Palli was born in Forlì, Italy, on January 6, 1977. He received the M.Sc. and Ph.D. degrees in automation engineering from the University of Bologna, Italy, in 2003 and 2007, respectively. He was a visiting student at the Robotic Institute of the German Aerospace Center (DLR), Munich, Germany, in 2006. Currently, he is an assistant professor at the University of Bologna. His research interests include the design and control of robotic hands, the modeling and control of robots with variable stiffness joints, the design of compliant structures and actuation systems for robotics applications and the development of real time systems for automatic control applications.

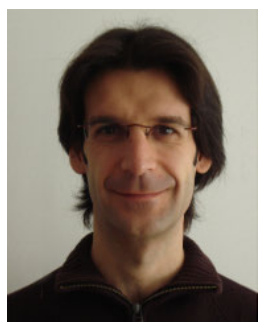

J. Javier Fernández received a B.Sc. in Industrial Electronics and Automation Engineering (2000), a M.Sc. in Automation and Industrial Computing (2003) both by the Polytechnic University of Valencia, Spain. Currently, he is a researcher at Interactive and Robotic Systems Laboratory (IRSLab) at the Jaume I University, Spain, where is performing a $\mathrm{Ph} . \mathrm{D}$. in Advanced Computer Systems. His research interests are underwater robotics, autonomous manipulation, mechatronics and control systems.

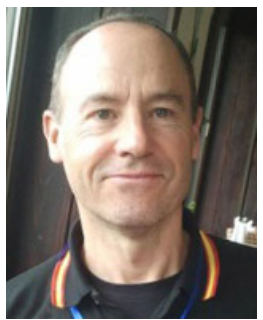

Pedro J. Sanz is Full Professor in the Computer Science and Engineering Department at Jaume I University (UJI, Spain), and head of the Interactive and Robotic Systems Lab. He holds a B.Sc. in Physics by the University of Valencia, M.Sc. in Engineering from the Technical University of Valencia and a Ph.D. in Computer Engineering by UJI. His current research interests are Multisensory based Grasping and Dexterous Manipulation, Telerobotics and Human-Robot Interaction, all of them applied to real scenarios, including assistive and underwater robotics. He is author or co-author of a broad range of research publications and is an active member of different scientific societies such as IEEE (RAS "\&" SMC), EUCog, and euRobotics; and served as As-Ed of Int. Journals (e.g. IEEE Tr. on SMC(C) and IEEE RAM). He has been appointed as Visiting Scientist at different research Labs in Europe: TUM (GERMANY, 2000), Blaise Pascal (FRANCE, 2002), Bologna (ITALY, 2008), and Girona (Spain, 2011). 